\title{
Are some patient-perceived migraine triggers simply early manifestations of the attack?
}

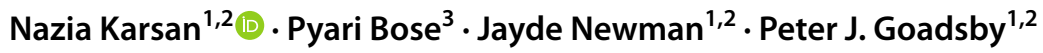

Received: 18 October 2020 / Revised: 21 November 2020 / Accepted: 4 December 2020 / Published online: 5 January 2021

(c) The Author(s) 2020

\begin{abstract}
Objective To study the agreement between self-reported trigger factors and early premonitory symptoms amongst a group of migraineurs in both spontaneous and pharmacologically provoked attacks.

Methods Fifty-three subjects with migraine with and without aura, with $\leq 22$ headache days/month, with spontaneous premonitory symptoms associated with migraine attacks were recruited nationally. A detailed history was taken by a study investigator to confirm diagnosis and extended phenotyping was performed to identify patient-reported triggers for migraine attacks, premonitory symptom phenotype and headache characteristics, using a standardised physician-administered questionnaire. The same subjects were exposed to a $0.5 \mathrm{mcg} / \mathrm{kg} / \mathrm{min}$ nitroglycerin infusion over $20 \mathrm{~min}$, to determine if similar migraine symptoms could be triggered. The triggered attacks were phenotyped in the same way as spontaneous ones. Percentage agreement and Cohen's kappa measure of agreement were used to identify concordance between patient-reported triggers and the corresponding spontaneous and triggered premonitory symptoms. Percentage agreement of $>60 \%$ and/or a kappa value $>0.3$ with $P<0.05$ were considered significant.

Results There was statistically significant agreement between perception of light as a migraine trigger and spontaneous premonitory photophobia; perception of sound as a trigger and triggered premonitory phonophobia; skipping meals as a trigger and spontaneous premonitory food cravings; and food triggers and spontaneous premonitory food cravings. There was good agreement between stress and premonitory triggered mood change.

Conclusions At least some patient-reported triggers, such as light, sound, foods and skipping meals, may represent early brain manifestations of the premonitory phase of the migraine attack.
\end{abstract}

Keywords Migraine $\cdot$ Premonitory $\cdot$ Prodrome $\cdot$ Triggers $\cdot$ Headache

\section{Introduction}

Migraine is a common and disabling disorder [1]. Despite considerable therapeutics research over several decades, not all patients are well served by migraine treatments $[2$, 3]. This can leave patients and physicians frustrated and

Nazia Karsan

nazia.karsan@kcl.ac.uk

1 Headache Group, Department of Basic and Clinical Neuroscience, Institute of Psychiatry, Psychology and Neuroscience, King's College London, London, UK

2 NIHR-Wellcome Trust King's Clinical Research Facility, SLaM Biomedical Research Centre, King's College Hospital, London, UK

3 Present Address: Department of Neurology, Auckland City Hospital, Auckland, New Zealand searching for other options for disease management. Many search for attack triggers as a preventive strategy and can make extensive dietary and lifestyle modifications to try to help their headache burden [4]. However, despite patient reports of a wide range of triggers in clinical practice, there is little objective evidence for commonly associated triggers, such as chocolate [5] and bright lights [6], reproducing migraine in experimental studies.

Increasingly recognised in migraine is the premonitory phase, the earliest phase of the attack, which is characterised by sensory sensitivities, food cravings and mood change, occurring hours to days prior to pain onset [7]. A range of prevalence of premonitory symptoms is reported in the literature, across varying study designs, with a general increase in prevalence reported with time [8-14], suggesting that perhaps increased attention to this phase, patient and physician education, and recognition of these 
symptoms have led to an increase in symptom identification. The lack of experimental evidence for many migraine triggers, and the generally poor response in migraine frequency to avoidance of such factors [15], has prompted a re-evaluation of whether patient-perceived migraine triggers are true triggers, or, in some part, incorrectly attributed early premonitory manifestations of the attack [16, 17]. It is feasible that if a patient experiences a symptom before migraine headache, such as a chocolate craving, they may then go and consume some chocolate, and then when they develop a headache some hours to a day later, assume that the chocolate triggered the headache, when in fact the migraine attack had likely already started within the brain.

Recently, functional neuroimaging studies have suggested that there is early engagement of various brain areas during the premonitory phase; namely the hypothalamus, thalamus, limbic areas, sensory processing cortex and brainstem regions [18-24]. These areas functionally correlate with commonly reported symptoms during this time, such as thalamus and sensory cortices with photophobia, hypothalamus with homeostatic dysregulation and altered arousal and limbic areas with mood and cognitive change. Alterations in homeostasis and arousal, sensory perception, and mood and cognition are reported as premonitory symptoms and triggers. Early brain changes mediating premonitory symptoms before headache may, therefore, lead to the correct association of these symptoms with corresponding trigger factors, but incorrect attribution that the trigger factor is directly causing the headache.

We wished to examine reported triggers and premonitory symptoms amongst the same individuals in patients with migraine with and without aura, to look for such an association in both retrospectively reported spontaneous attacks, and prospectively observed nitroglycerin-triggered attacks. The latter, in case subjects had not themselves noticed particular premonitory symptoms associated with spontaneous attacks. The range of prevalence of these symptoms reported in the literature suggest that under-recognition is likely to be an issue at least for some patients. Nitroglycerin (NTG) has been shown to be able to trigger both premonitory symptoms and migraine headache, thus making it a suitable experimental migraine model for this study [25]. In addition, the phenotype of premonitory symptoms following nitroglycerin has been shown to be comparable to those following spontaneous attacks in the same individual [26].

\section{Methods}

The methodology used in this study has been previously reported [26] and is summarised here.

\section{Subject recruitment}

Subjects with migraine were identified through online advertisements, bulletins, patient group advertising and university advertising and through local and national headache clinics. The inclusion criteria for the study included a diagnosis of migraine with or without aura, using ICHD-3 beta, which was in use at the time of the study [27]. We identified subjects with up to 22 headache days a month, a history of spontaneous premonitory symptoms with attacks, and no medical or psychiatric contraindications to study participation and/or NTG exposure. Use of any oral single agent oral preventive therapy for migraine was allowed. Exclusion criteria included medication overuse, illicit drug use, excess alcohol consumption and smoking. Use of more than one oral preventive agent for migraine, the use of neuromodulatory devices, or both, and onabotulinum toxin type A and/or greater occipital nerve injections within the past 3 months were also excluded.

Given the generally poor ability of NTG to trigger aura [28-30], we did not anticipate that aura would be triggered in the study and act as a potential confound to identification of premonitory symptoms. We included subjects on single agent preventive therapy to aid recruitment, noting that it has never been systematically studied if the use of migraine preventive agents alters the reporting of premonitory symptoms. Recruitment was completed from February 2015 to July 2017.

\section{Sample size}

The initial nitroglycerin triggering study, from which these data are extracted, aimed to study sufficient subjects exposed to nitroglycerin to exceed our previous experience [25]. We, therefore, aimed to expose at least 50 subjects to nitroglycerin.

\section{Screening}

Three hundred and fifty subjects were pre-screened for eligibility. Of these 350 subjects, 53 (15\%) met eligibility criteria, agreed to attend a screening visit and were included in the final analysis. There was a large pre-screening failure rate, mostly due to too frequent headache and use of more than one migraine preventive agent.

All study visits were performed within the Clinical Research Facility at King's College Hospital, London, UK.

A prior history of headache or any migraine symptoms or treatment was not allowed $12 \mathrm{~h}$ prior to the study visit. The screening visit involved written consent for study participation, followed by detailed phenotyping of spontaneous 
migraine attacks, triggers, medication history and ensuring no medical or pharmaceutical contraindications to any of the study drugs, including nitroglycerin and acute migraine treatments used to treat pain in the study: intravenous aspirin and subcutaneous sumatriptan. Each symptom or trigger was reported as a yes/no answer, and all positive responses were recorded, including the reporting of multiple triggers. An appropriate cardiovascular and neurological examination was performed to exclude obvious cardiac contraindications to study participation and secondary cause for a headache disorder. An ECG was performed to exclude cardiac contraindications to nitroglycerin or triptan exposure. The spontaneous migraine attacks were retrospectively phenotyped in detail, using a physician-administered symptom questionnaire (Fig. 1). Subjects were allowed to volunteer any triggers or symptoms not mentioned in the questionnaire.

\section{Nitroglycerin triggering}

Following the history and examination, each subject was exposed to a $0.5-\mathrm{mcg} / \mathrm{kg} / \mathrm{min}$ nitroglycerin infusion over $20 \mathrm{~min}$. Subjects were symptomatically and haemodynamically assessed with blood pressure, heart rate, and oxygen

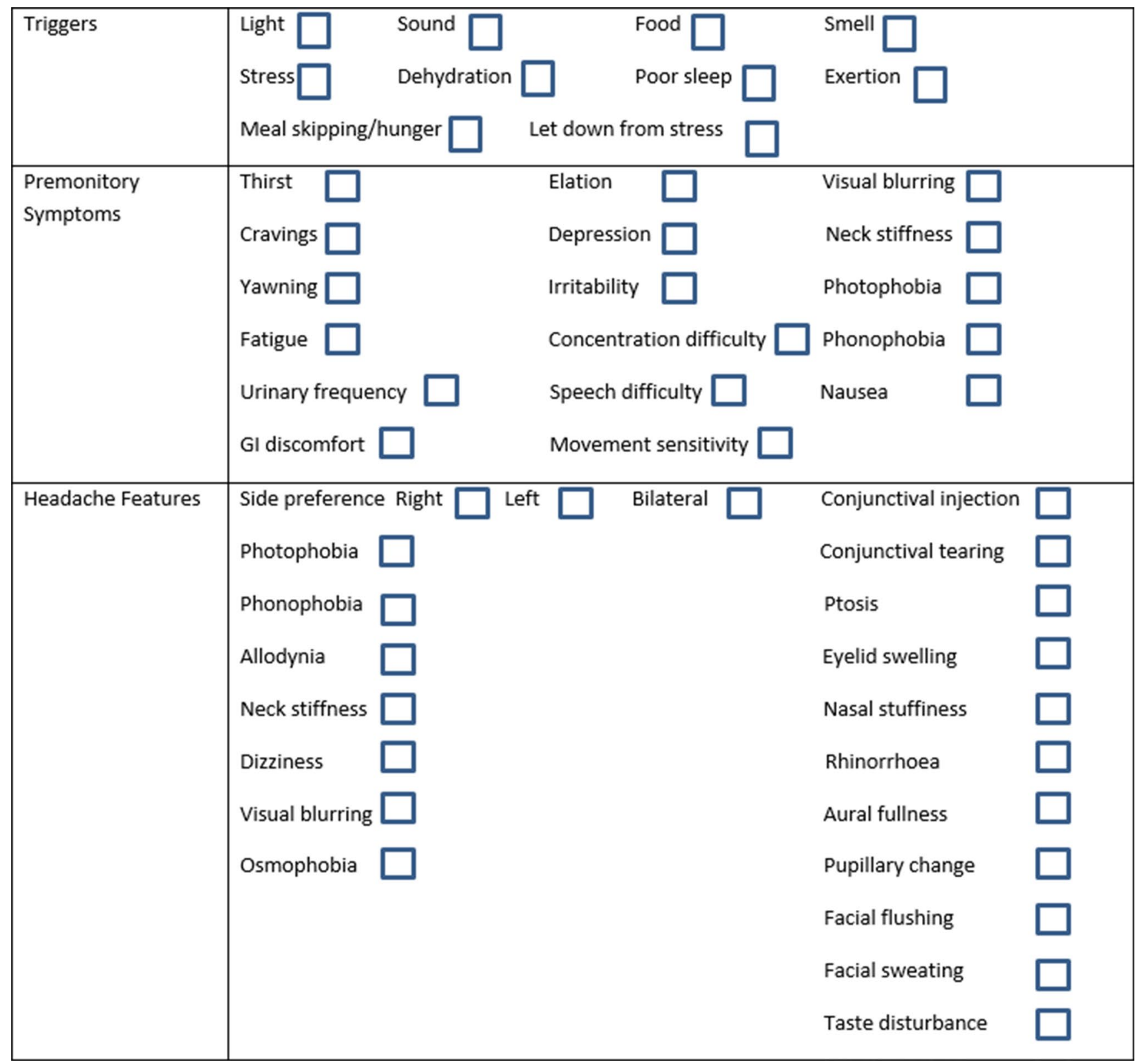

Fig. 1 Phenotypic data collection questionnaire for both spontaneous and triggered attacks 
saturation monitoring before the infusion and at 5-min intervals during the infusion. Each subject was prospectively questioned regarding the evolution of any headache, its site, severity, phenotype and the presence of any other symptoms, including typical premonitory symptoms using the same physician-administered symptom questionnaire that was used for spontaneous attacks (Fig. 1). Questioning continued at 15-min intervals following the infusion until the time of headache resolution following treatment. The answers to the premonitory symptom question presence were binary (yes/no), without any further grading of intensity or severity.

A premonitory symptom was defined as any symptom that the patient experienced following nitroglycerin infusion in the absence of migraine headache, which was typical for a symptom they would experience prior to a spontaneous migraine headache.

Nitroglycerin is a drug agent with vasodilatory actions and a short half-life (minutes) [31]. Within the brain, the vasodilatory drug effects are thought to peak at a similar time, and possibly last for no more than 45 min $[32,33]$. Drug side effects typically come on quickly after intravenous administration and usually resolve within 5-10 min. We would, therefore, hope that with the design of the study, drug effects, if any, would be minimal or absent during premonitory symptoms.

Whilst our previous work has looked at symptoms triggered across serial nitroglycerin exposures and following placebo [26], for this study, we aimed to compare only spontaneous and nitroglycerin-triggered symptoms on the first nitroglycerin exposure.

\section{Treatment of triggered attacks}

All delayed migrainous headache following nitroglycerin was treated as soon as it reached moderate-severe intensity, using either $1 \mathrm{~g}$ intravenous aspirin or $6 \mathrm{mg}$ subcutaneous sumatriptan, based on subjects' usual response. Headache freedom was necessary before a subject could be discharged from the Research Facility.

\section{Statistical analysis}

Percentage agreement between spontaneous trigger factor and spontaneous and triggered reporting of the corresponding premonitory symptom and Cohen's kappa analysis were used to assess agreement between selected trigger factors and corresponding premonitory symptoms. For subjects who reported more than one spontaneous trigger, each trigger factor was analysed for association with the corresponding premonitory symptom separately. The groups were selected based on feasibility of the triggers and premonitory symptoms being associated:
Light trigger-Premonitory photophobia.

Sound trigger-Premonitory phonophobia.

Stress trigger-Premonitory mood change.

Stress trigger-—Premonitory neck stiffness.

Food trigger-Premonitory food cravings.

Dehydration trigger-Premonitory thirst.

Hunger/skipping meals trigger-Premonitory food crav-

ings.

Poor sleep trigger-Premonitory fatigue.

All of these associations were tested for both spontaneous and nitroglycerin-triggered attacks. SPSS v 24 was used for all statistical analyses. Percentage agreement was calculated using a $2 \times 2$ crosstabulation of yes/no results for trigger and premonitory symptom and taking a sum of the yes/yes and no/no responses. Percentage agreement of $>60 \%$ and/or a kappa value of $>0.3$ (fair-good agreement) with $P<0.05$ (kappa significantly different to 0 ) was considered significant, as using kappa alone can have its limitations when assessing agreement between dichotomous variables [34].

\section{Results}

\section{Subject demographics}

Of the 53 subjects, nine were males. Twenty-seven had migraine with aura (51\%), 20 had migraine without aura (38\%) and 6 had chronic migraine (11\%). Sixteen (30\%) were on single agent preventive therapy. The age range of subjects was 18-50 years (mean 36 years), with up to 22 headache days per month (median 8 days, range 1-22 days). Subject demographics are summarised in Table 1.

\section{Nitroglycerin triggering}

Forty-four subjects (83\%) developed delayed migrainous headache following the nitroglycerin infusion (range 20-278 min following nitroglycerin, median $107 \mathrm{~min}$ ), and all but one (98\%) had at least one typical premonitory symptom preceding the headache (range 4-155 min, median $23 \mathrm{~min}$ ). Typical aura symptoms were triggered in 7 of the 53 subjects (13\%). The aura phenotypes were visual aura (one was not followed by delayed migraine), hemisensory, hemimotor and Alice in Wonderland syndrome.

\section{Spontaneous triggers and spontaneously reported premonitory symptoms}

The results for spontaneously reported triggers and spontaneous premonitory symptoms are shown in Table 2 . The significant results are highlighted in bold. 
Table 1 Summary of subject demographics $(n=53)$. NSAID: non-steroidal anti-inflammatory drug

$\begin{array}{ll}\text { Age } & 18-50 \text { years, mean 36 years } \\ \text { Sex } & 9 \text { males, } 44 \text { females } \\ \text { Baseline headache days per month (range, median) } & 0-22 \text { days, median } 8 \text { days } \\ \text { Baseline headache diagnosis } & 27 \text { Episodic migraine with aura } \\ & \text { 20 Episodic migraine without aura } \\ \text { Preventive use }(n=16) & 7 \text { Chronic migraine } \\ & \text { Beta-blocker-6; amitriptyline-3; } \\ & \text { topiramate-3; pizotifen-2, cande- } \\ \text { Acute abortive treatment } & \text { sartan-2 } \\ & \text { Non-steroidal anti-inflammatory 11 } \\ & \text { Triptan 25 } \\ & \text { NSAID/triptan combination 2 } \\ & \text { Paracetamol/NSAID combination 10 } \\ \text { Baseline MIDAS score (range, median, IQR) } & \text { Paracetamol/codeine combination 5 } \\ \end{array}$

Table 2 Agreement analysis between spontaneously reported triggers and corresponding premonitory symptoms

\begin{tabular}{|c|c|c|c|c|c|c|}
\hline $\begin{array}{l}\text { Subject-reported trig- } \\
\text { ger factor }\end{array}$ & $\begin{array}{l}\text { Correspond- } \\
\text { ing premonitory } \\
\text { symptom }\end{array}$ & $\begin{array}{l}\text { Number report- } \\
\text { ing trigger factor } \\
(n)\end{array}$ & $\begin{array}{l}\text { Number reporting } \\
\text { spontaneous premoni- } \\
\text { tory symptom }(n)\end{array}$ & $\begin{array}{l}\text { Percentage agreement } \\
\text { spontaneous attacks } \\
(\%)\end{array}$ & $\begin{array}{l}\text { Kappa } \\
\text { spontaneous } \\
\text { attacks }\end{array}$ & $\begin{array}{l}P \text { value spon- } \\
\text { taneous attacks }\end{array}$ \\
\hline Light & Photophobia & 24 & 12 & 75 & 0.3 & 0.03 \\
\hline Sound & Phonophobia & 12 & 7 & 29 & 0.05 & 0.7 \\
\hline Stress & Mood change & 26 & 37 & 46 & 0 & 0.5 \\
\hline Stress & Neck stiffness & 26 & 30 & 53 & 0.01 & 0.5 \\
\hline Food & Cravings & 17 & 3 & 59 & 0.4 & 0.004 \\
\hline Dehydration & Thirst & 10 & 18 & 22 & 0.06 & 0.7 \\
\hline $\begin{array}{l}\text { Hunger/skipping } \\
\text { meals }\end{array}$ & Cravings & 7 & 17 & 29 & 0.3 & 0.02 \\
\hline Poor sleep & Fatigue & 13 & 41 & 22 & 0 & 0.4 \\
\hline
\end{tabular}

The most commonly reported trigger was stress (stress and let-down from stress) $(n=26)$, followed by light $(n=24)$ and certain foods $(n=17)$. The most common premonitory symptoms reported during spontaneous attacks were concentration difficulty $(n=44)$, fatigue $(n=41)$, mood change $(n=34)$ and neck stiffness $(n=28)$. Between 1 and 9 premonitory symptoms were reported during spontaneous attacks (median 5, IQR 4-7). There was good agreement for the reporting of light as a trigger and spontaneous premonitory photophobia, food as a trigger and premonitory cravings and hunger or skipping meals as a trigger and premonitory cravings.

\section{Spontaneous triggers and triggered premonitory symptoms reported}

The results for spontaneously reported triggers and triggered premonitory symptoms are shown in Table 3 . The significant results are highlighted in bold.

The most common premonitory symptoms reported during nitroglycerin-triggered attacks were fatigue $(n=43)$, photophobia $(n=35)$, concentration difficulty $(n=34)$ and neck stiffness $(n=32)$. Between 0 and 9 premonitory symptoms were triggered with nitroglycerin (median 5 , IQR 3-6). There was good agreement for the reporting of light as a trigger and nitroglycerin-triggered premonitory photophobia, sound as a trigger and nitroglycerintriggered phonophobia and stress as a trigger and nitroglycerin-triggered premonitory mood change.

The frequency of reporting different trigger factors and associated premonitory symptoms during spontaneous (blue) and nitroglycerin-triggered (orange) migraine attacks are summarised in Fig. 2.

There are some differences in the associations between spontaneous and nitroglycerin-triggered attacks, which one could attribute to the environmental differences during spontaneous attacks and study visits, as well as increased patient recognition during direct observation of attacks and questioning on symptoms compared to retrospective recall of spontaneous attacks. This included increased recognition of symptoms like photophobia, phonophobia and movement sensitivity. 
Table 3 Agreement analysis between spontaneously reported triggers and nitroglycerin-triggered premonitory symptoms

\begin{tabular}{|c|c|c|c|c|c|c|}
\hline $\begin{array}{l}\text { Subject-reported } \\
\text { trigger factor }\end{array}$ & $\begin{array}{l}\text { Corresponding } \\
\text { NTG-triggered pre- } \\
\text { monitory symptom }\end{array}$ & $\begin{array}{l}\text { Number report- } \\
\text { ing trigger factor } \\
(n)\end{array}$ & $\begin{array}{l}\text { Number reporting } \\
\text { NTG-triggered pre- } \\
\text { monitory symptom } \\
(n)\end{array}$ & $\begin{array}{l}\text { Percentage agree- } \\
\text { ment NTG-triggered } \\
\text { attacks (\%) }\end{array}$ & $\begin{array}{l}\text { Kappa NTG- } \\
\text { triggered } \\
\text { attacks }\end{array}$ & $\begin{array}{l}P \text { value NTG- } \\
\text { triggered attacks }\end{array}$ \\
\hline Light & Photophobia & 24 & 35 & 63 & 0.5 & $<0.001$ \\
\hline Sound & Phonophobia & 12 & 11 & 64 & 0.5 & $<0.001$ \\
\hline Stress & Mood change & 26 & 14 & 64 & 0.17 & 0.18 \\
\hline Stress & Neck stiffness & 26 & 32 & 50 & 0.02 & 0.9 \\
\hline Food & Cravings & 17 & 3 & 33 & 0 & 1 \\
\hline Dehydration & Thirst & 10 & 21 & 19 & 0 & 1 \\
\hline $\begin{array}{l}\text { Hunger/skipping } \\
\text { meals }\end{array}$ & Cravings & 7 & 3 & 33 & 0.13 & 0.3 \\
\hline Poor sleep & Fatigue & 13 & 14 & 23 & 0 & 0.7 \\
\hline
\end{tabular}

Fig. 2 Summary of the percentage agreement between trigger factors and corresponding premonitory symptom in both spontaneous (blue) and nitroglycerin-triggered (orange) attacks [*Percentage agreement of $>60 \%$ and/or a kappa value of $>0.3$ (fair-good agreement) with $P<0.05$ ]

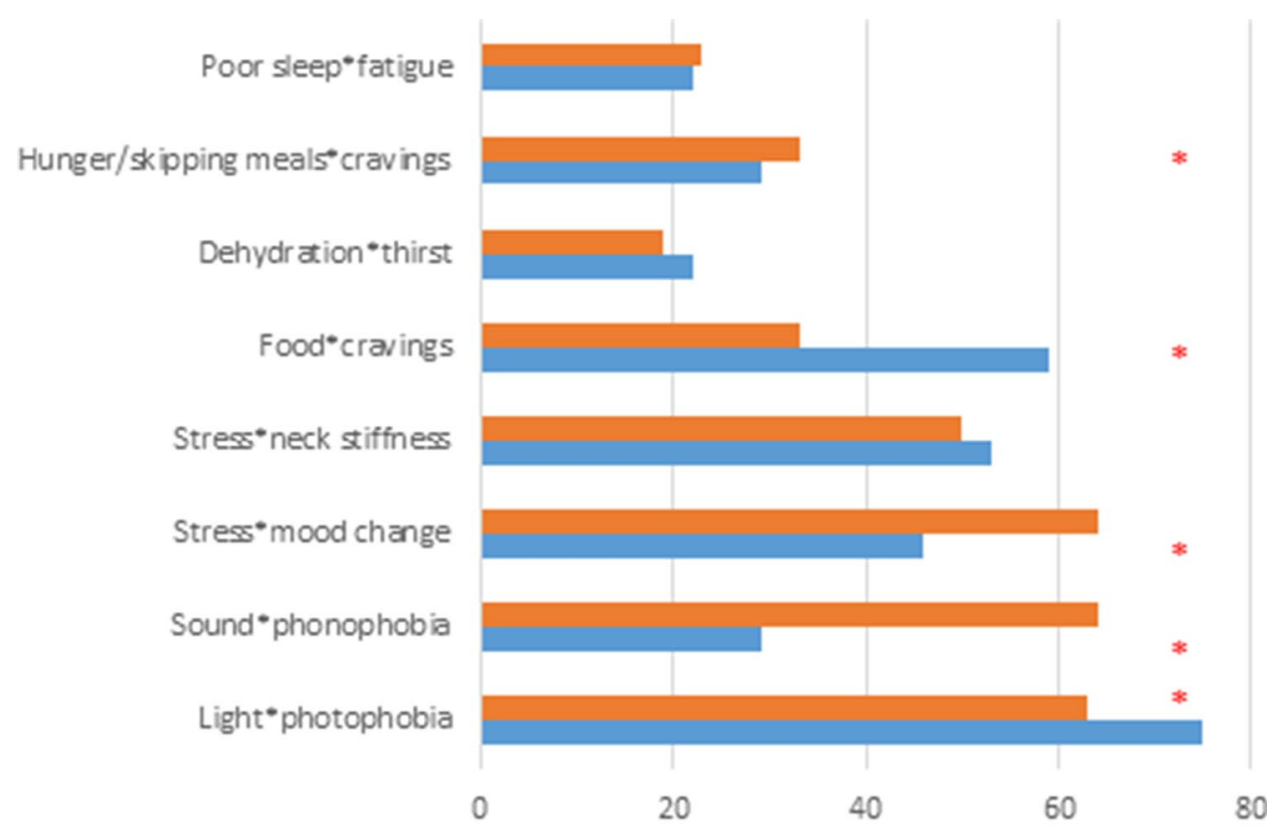

n Percentage agreement nitroglycer in-tr ggered atacks

n Percentage agreement spontaneous atacks

\section{Discussion}

Our results show an association between some spontaneous patient-reported triggers, and spontaneous and nitroglycerintriggered premonitory symptoms within the same subject. The findings are consistent with some reported triggers being premonitory symptoms associated with migraine that are mis-attributed by patients. Dissecting this relationship will help patients understand their own symptoms, and researchers build a better model of this complex neurological disorder.

During both spontaneous and triggered attacks, there was a significant association between the development of photophobia and light perceived as a trigger. There was discrepancy between spontaneous and triggered attacks for sound and phonophobia (triggered attacks only), stress and mood change (triggered attacks only), food and cravings and hunger and cravings (spontaneous attacks only). These differences are likely somewhat related to under-recognition of some premonitory symptoms by patients and, therefore, under-reporting in spontaneous attacks, as well as reporting bias caused by direct observation of triggered attacks. For example, it is feasible that mood change in the absence of a collateral witness may not be readily noticed by patients but could well have been noted by the physician during observation of triggered attacks. Similarly, food cravings were much 
less reported with triggered attacks than spontaneously, likely owing to environmental factors and lack of appetite stimulation during the triggered visit.

Whilst we have previously shown a comparable phenotype of spontaneous and nitroglycerin-triggered migraine attacks [26], it is clear that for some symptoms, there is a discrepancy, namely photophobia being more commonly triggered than reported spontaneously, and mood change being much more commonly reported spontaneously than during triggered attacks. In addition, the timeline of a nitroglycerin-triggered migraine attack is much shorter and generally compressed relative to a spontaneous attack. Triggered premonitory symptoms are typically followed by headache within $2 \mathrm{~h}$ [26], which is substantially less than what is seen in spontaneous attacks.

\section{Limitations and scope for further work}

Clearly the relationship between what may be a perceived trigger for a migraine attack and certain premonitory symptoms is complex and unlikely to be unidirectional. Migraine is a disorder influenced by genetic and epigenetic factors. Migraine is prone to change depending on both endogenous (such as hormonal change) and exogenous factors (such as sleep, alcohol and stress levels) [35]. We did not, therefore, expect to find an association between all the triggers that we captured and their corresponding premonitory symptoms. In addition, the use of different methods of data collection (retrospective and prospective), and the environment associated with the triggering visits, may have impacted on premonitory symptom phenotype. Going forwards, use of electronic diary systems and device application technology, following patient education, to allow prospective recording of perceived triggers, premonitory symptoms and their association to headache during spontaneous attacks, would be a valuable means of assessing these relationships, understanding better the mechanisms behind attack initiation and advancing therapeutics. Either way, increased patient education and recognition of the heterogeneity of migraine and the systematic recording of factors such as perceived triggers and early painless symptoms is likely to improve our understanding of the disorder. Vital questions behind how and why a migraine attack starts are being advanced by neuroimaging such that pre-pain phase treatments are a reasonable prospect in the future. In an era where despite the emergence of novel and targeted migraine therapies, there remains a need for effective attack abortion for many sufferers, such an understanding is likely to contribute to novel advances.

It is also important to highlight that whilst we compared triggers and premonitory symptoms in spontaneous and nitroglycerin-triggered attacks, prospective observation of triggered attacks is arguably not the same as prospective observation of spontaneous attacks, which would be ideal.
As discussed above, the differences in symptom capture and, therefore, association with triggers may have been in some part impacted on by nitroglycerin itself, or the environment in which it was administered, or indeed by the reporting bias induced by direct observation and questioning by a physician. Examining both spontaneous and triggered attacks in this subject cohort offered a reasonable starting point. Whilst we do not consider that there are likely to be fundamental biological differences between pharmacologically -provoked and spontaneously occurring migraine attacks (akin to alcohol-induced migraine attacks), the drug itself, the study environment and the recording of triggered symptoms may have impacted on the results and caused the observed differences in premonitory symptom association with triggers between spontaneous and triggered attacks. Spontaneous attacks occur in patients' own surroundings (home, work, school) and most often occur in the absence of an exogenous trigger. Patients self-report their symptoms, whilst nitroglycerin-triggered attacks are provoked, are directly observed within a clinical facility and the phenotype of symptoms reported thereafter is captured both through patient selfreporting and through direct questioning by a physician. These differences are important to recognise, and highlight the importance of large-scale population-based prospective diary studies going forwards, to further inform on these relationships in the future.

\section{Conclusions}

Despite the limitations discussed, there does seem to be a relationship between some triggers and premonitory symptoms. Such a misperception would be consistent with the generally poor outcomes of trigger studies involving putative agents, such as chocolate and light, and with premonitory phase biology driving behaviours that are correctly associated with the trigger factors yet falsely attributed to them. Emerging functional imaging work, as well as animal migraine models looking at some premonitory symptoms, has suggested that early activation of brain areas, pathways and neuropeptide systems occur during the premonitory phase and are responsible for mediating some of these symptoms via hypothalamic and other diencephalic mechanisms [36]. There is, therefore, a neuroimaging correlate for how these symptoms may be mediated centrally, for example, with the thalamus and connections to visual cortex likely being involved in photophobia and hypothalamic connections to pontine areas and pontine connections to cortical areas being involved in regulation of feeding [24].

Going forwards, systematic and prospective attack recording is likely to be useful in advancing neuroimaging work and therapeutics. 
Acknowledgements The authors are grateful to the funders and participants who were involved in this study, as well as the NIHR-Wellcome Trust King's Clinical Research Facility staff for their support and contribution.

Author contributions NK: Main author and study investigator (study protocol, ethical approval, study conduct, data collection, data collation, statistical analysis, interpretation, writing of manuscript). PRB: Study co-investigator (study protocol, ethical approval, study conduct, data collection). JN: Data collation, statistical analysis. PJG: Senior author and principal investigator (study protocol, ethical approval, review of data analysis and manuscript prior to submission).

Funding This study was partly funded by an Association of British Neurologists and Guarantors of Brain Clinical Research Training Fellowship awarded to Dr. Karsan, and a Migraine Trust Clinical Research Fellowship awarded to Dr. Bose. This study represents independent research part-funded by the National Institute for Health Research (NIHR) Biomedical Research Centre at South London and Maudsley NHS Foundation Trust and King's College London. The views expressed are those of the authors, and not necessarily those of the NHS, the NIHR or the Department of Health and Social Care.

Data availability The principal author had full access to those data and has maintained the right to publish any and all data independent of any third party. The data (anonymised) and full study protocol are available at request.

\section{Compliance with ethical standards}

Conflicts of interest The authors report no relevant conflicts of interest related to this work. Separate to this work, NK has received honoraria from Novartis Pharmaceuticals and Teva Pharmaceuticals, and writer honoraria from Continuum and Medicine (MPMED). PB has received honoraria from Teva Pharmaceuticals and writer honoraria from Continuum. JN has no disclosures to report. PJG reports, over the last 36 months, grants and personal fees from Amgen and Eli-Lilly and Company, grant from Celgene, and personal fees from Alder Biopharmaceuticals, Aeon Biopharma, Allergan, Biohaven Pharmaceuticals Inc., Clexio, Electrocore LLC, eNeura, Epalex, GlaxoSmithKline, Impel Neuropharma, Lundbeck, MundiPharma, Novartis, Pfizer, Sanofi, Santara Therapeutics, Teva Pharmaceuticals, Trigemina Inc., WL Gore, and personal fees from MedicoLegal work, Massachusetts Medical Society, Up-to-Date, Oxford University Press, and Wolters Kluwer; and a patent magnetic stimulation for headache assigned to eNeura without fee.

Ethics approval This study using human participants was approved by the Camden and King's Cross Research Ethics Committee in February 2015 (14/LO/2241). The study was also approved by King's College Hospital Research and Development Committee.

Consent to participate All subjects enrolled in the study gave informed written consent for participation, according to the Declaration of Helsinki.

Open Access This article is licensed under a Creative Commons Attribution 4.0 International License, which permits use, sharing, adaptation, distribution and reproduction in any medium or format, as long as you give appropriate credit to the original author(s) and the source, provide a link to the Creative Commons licence, and indicate if changes were made. The images or other third party material in this article are included in the article's Creative Commons licence, unless indicated otherwise in a credit line to the material. If material is not included in the article's Creative Commons licence and your intended use is not permitted by statutory regulation or exceeds the permitted use, you will need to obtain permission directly from the copyright holder. To view a copy of this licence, visit http://creativecommons.org/licenses/by/4.0/.

\section{References}

1. GBD 2016 Headache Collaborators (2018) Global, regional, and national burden of migraine and tension-type headache, 1990 2016: a systematic analysis for the Global Burden of Disease Study 2016. Lancet Neurol 17(11):954-976

2. Lipton RB et al (2019) Unmet acute treatment needs from the 2017 migraine in America symptoms and treatment study. Headache 59(8):1310-1323

3. Hepp Z, Bloudek LM, Varon SF (2014) Systematic review of migraine prophylaxis adherence and persistence. J Manag Care Pharm 20(1):22-33

4. Pellegrino ABW et al (2018) Perceived triggers of primary headache disorders: a meta-analysis. Cephalalgia 38(6):1188-1198

5. Moffett AM, Swash M, Scott DF (1974) Effect of chocolate in migraine: a double-blind study. J Neurol Neurosurg Psychiatry $37(4): 445-448$

6. Hougaard A et al (2013) Provocation of migraine with aura using natural trigger factors. Neurology 80(5):428-431

7. Giffin NJ et al (2003) Premonitory symptoms in migraine: an electronic diary study. Neurology 60(6):935-940

8. Laurell $\mathrm{K}$ et al (2016) Premonitory symptoms in migraine: a cross-sectional study in 2714 persons. Cephalalgia 36(10):951-959

9. Blau JN (1980) Migraine prodromes separated from the aura: complete migraine. Br Med J 281(6241):658-660

10. Russell MB et al (1996) Migraine without aura and migraine with aura are distinct clinical entities: a study of four hundred and eighty-four male and female migraineurs from the general population. Cephalalgia 16(4):239-245

11. Rasmussen BK, Olesen J (1992) Migraine with aura and migraine without aura: an epidemiological study. Cephalalgia 12(4):221-228

12. Schoonman GG et al (2006) The prevalence of premonitory symptoms in migraine: a questionnaire study in 461 patients. Cephalalgia 26(10):1209-1213

13. Kelman L (2004) The premonitory symptoms (prodrome): a tertiary care study of 893 migraineurs. Headache 44(9):865-872

14. Drummond PD, Lance JW (1984) Neurovascular disturbances in headache patients. Clin Exp Neurol 20:93-99

15. Martin PR et al (2014) Behavioral management of the triggers of recurrent headache: a randomized controlled trial. Behav Res Ther 61:1-11

16. Schulte LH, Jurgens TP, May A (2015) Photo-, osmo- and phonophobia in the premonitory phase of migraine: mistaking symptoms for triggers? J Headache Pain 16:14

17. Pavlovic JM et al (2014) Trigger factors and premonitory features of migraine attacks: summary of studies. Headache 54(10):1670-1679

18. Maniyar FH et al (2014) Brain activations in the premonitory phase of nitroglycerin-triggered migraine attacks. Brain $137(\mathrm{Pt}$ 1):232-241

19. Schulte L et al (2018) Brain networks in migraine: continuous resting state fMRI over 30 days. Cephalalgia 38(1S):32-33

20. Schulte LH, May A (2016) The migraine generator revisited: continuous scanning of the migraine cycle over 30 days and three spontaneous attacks. Brain 139(Pt 7):1987-1993 
21. Schulte LH, Meynert J, May A (2020) Longitudinal neuroimaging over 30 days: temporal characteristics of migraine. Annals Neurol 87(4):646-651

22. Marciszewski KK et al (2018) Changes in brainstem pain modulation circuitry function over the migraine cycle. J Neurosci 38(49):10479-10488

23. Meylakh $\mathrm{N}$ et al (2018) Deep in the brain: changes in subcortical function immediately preceding a migraine attack. Hum Brain Mapp 39(6):2651-2663

24. Karsan $\mathrm{N}$ et al (2020) Alterations in functional connectivity during different phases of the triggered migraine attack. Headache 60(7):1244-1258

25. Afridi SK, Kaube H, Goadsby PJ (2004) Glyceryl trinitrate triggers premonitory symptoms in migraineurs. Pain 110(3):675-680

26. Karsan $\mathrm{N}$ et al (2020) Headache and non-headache symptoms provoked by nitroglycerin in migraineurs: a human pharmacological triggering study. Cephalalgia 40(8):828-841

27. Headache Classification Committee of the International Headache Society (2013) The international classification of headache disorders, 3rd edition (beta version). Cephalalgia 33(9):629-808

28. Christiansen I et al (1999) Glyceryl trinitrate induces attacks of migraine without aura in sufferers of migraine with aura. Cephalalgia 19(7):660-667
29. Hansen JM et al (2008) Familial hemiplegic migraine type 1 shows no hypersensitivity to nitric oxide. Cephalalgia 28(5):496-505

30. Hansen JM et al (2008) Familial hemiplegic migraine type 2 does not share hypersensitivity to nitric oxide with common types of migraine. Cephalalgia 28(4):367-375

31. Abrams J (1985) Pharmacology of nitroglycerin and long-acting nitrates. Am J Cardiol 56(2):12a-18a

32. Schoonman GG et al (2008) Migraine headache is not associated with cerebral or meningeal vasodilatation - a 3T magnetic resonance angiography study. Brain 131(Pt 8):2192-2200

33. Hansen JM et al (2007) Magnetic resonance angiography shows dilatation of the middle cerebral artery after infusion of glyceryl trinitrate in healthy volunteers. Cephalalgia 27(2):118-127

34. Feinstein AR, Cicchetti DV (1990) High agreement but low kappa: I. The problems of two paradoxes. J Clin Epidemiol 43(6):543-549

35. Serrano D et al (2017) Fluctuations in episodic and chronic migraine status over the course of 1 year: implications for diagnosis, treatment and clinical trial design. J Headache Pain 18(1):101

36. Karsan N, Goadsby PJ (2018) Biological insights from the premonitory symptoms of migraine. Nat Rev Neurol 14(12):699-710 